\title{
Point-of-care training program on COVID-19 infection prevention and control for pediatric healthcare workers: a multicenter, cross- sectional questionnaire survey in Shanghai, China
}

\author{
Yingzi Ye ${ }^{1,2}$, Peng Shi ${ }^{1} \wedge$, Yonghao Gui ${ }^{2,3}$, Albert M. Li ${ }^{4}$, Guoying Huang ${ }^{1,2}$, Hong Xu ${ }^{1,2}$, Quan Lu ${ }^{2,5}$, \\ Jianguo Hong ${ }^{2,6}$, Ying $\mathrm{Gu}^{1}$, Xiaojing $\mathrm{Hu}^{1}$, Gongbao Liu ${ }^{1}$, Chuanqing Wang ${ }^{1}$, Qin Huang ${ }^{1}$, Xiaobo Zhang ${ }^{1,2} \wedge$ \\ ${ }^{1}$ Children's Hospital of Fudan University, National Children's Medical Center, Shanghai, China; ${ }^{2}$ Center for Pediatric Clinical Quality Control of \\ Shanghai, Shanghai, China; ${ }^{3}$ Shanghai Medical College, Fudan University, Shanghai, China; ${ }^{4}$ Department of Pediatrics, The Chinese University of \\ Hong Kong, Hong Kong, China; ${ }^{5}$ Department of Respiratory Disease, Children's Hospital of Shanghai, Shanghai Jiao Tong University, Shanghai, \\ China; ${ }^{6}$ Department of Pediatrics, Shanghai General Hospital, Shanghai Jiao Tong University, Shanghai, China \\ Contributions: (I) Conception and design: X Zhang, Y Ye, P Shi; (II) Administrative support: X Zhang, Y Ye, Y Gui, AM Li; (III) Provision of study \\ materials or patients: G Huang, H Xu, Q Lu, J Hong, Y Gui, X Hu, G Liu, C Wang, Q Huang; (IV) Collection and assembly of data: Y Ye, P Shi, G \\ Huang, H Xu, Q Lu, J Hong, Y Gui, X Hu, G Liu, C Wang, Q Huang; (V) Data analysis and interpretation: Y Ye, P Shi; (VI) Manuscript writing: \\ All authors; (VII) Final approval of manuscript: All authors. \\ Correspondence to: Xiaobo Zhang. Professor of Pediatrics, Children's Hospital of Fudan University, 399 Wan Yuan Road, Shanghai 201102, China. \\ Email: xiaobozhang@fudan.edu.cn.
}

Background: To evaluate the effectiveness of training on knowledge and practice of infection prevention and control (IPC) among pediatric health care workers (HCW) in Shanghai, China, in the context of COVID-19 pandemic.

Methods: An online training program was designed by the Shanghai Pediatric Clinical Quality Control Center (SPQCC) during the early phase of COVID-19 pandemic on disease knowledge and practice of IPC. Training took place in the 81 partner hospitals affiliated with SPQCC. A multicenter, crosssectional questionnaire survey was designed with a 25 -item self-administered questionnaire to evaluate the knowledge gained from the training. Stratified-random sampling was used to select HCW according to three professionals (i.e., pediatricians, nurses and administrators) within each partner hospital. Awareness and knowledge of COVID-19 and its related infection control and practice were assessed by comparing survey results between different types of hospitals, professionals and professional ranks. A higher survey score meant that the respondent was more prepared and knowledgeable about COVID-19 and its infection control measures.

Results: Completed questionnaires were returned from 1,062 subjects (385 pediatricians, 410 nurses, and 267 administrators), giving a response rate of $96.5 \%$. Overall, awareness of clinical information related to COVID-19, importance of personal hygiene and isolation policy was high among the respondents. No statistical difference of scores on knowledge of COVID-19, IPC and relevant practice between the tertiary and peripheral hospitals. Among all respondents, middle-ranked health care personnel were most knowledgeable and achieved the highest score.

Conclusions: Majority of pediatric HCW showed good recognition and practice in infection protection and control measures. The online training was able to achieve its aim to enhance knowledge and awareness and could have contributed to the zero infection rate among HCW caring for confirmed COVID-19 cases in Shanghai.

^ ORCID: Peng Shi, 0000-0002-0626-4555; Xiaobo Zhang, 0000-0002-7666-9638. 
Keywords: Awareness; infection prevention and control (IPC); pediatric healthcare workers; COVID-19; survey

Submitted Aug 18, 2020. Accepted for publication Nov 30, 2020.

doi: $10.21037 / \mathrm{tp}-20-247$

View this article at: http://dx.doi.org/10.21037/tp-20-247

\section{Introduction}

The emergence of severe acute respiratory syndrome coronavirus 2 (SARS-CoV-2; previously provisionally named 2019 novel coronavirus or $2019-\mathrm{nCoV}$ ) disease (COVID-19) at the end of 2019 has become a worldwide epidemic. An important public health problem, COVID-19 is highly infectious and can lead to significant mortality in the elderly and high-risk groups (1-5). So far, it has affected around $21,260,000$ persons in 216 countries/ regions with more than 761,000 deaths reported (6).

Studies have confirmed human-to-human transmission of SARS-CoV-2 (2). The predominant mechanism of transmission is thought to be contact/droplet spread related to respiratory secretions (7). Isolated reports detecting SARS-CoV-2 in stool have also raised concerns about the potential risk of fecal-oral transmission (8). Moreover, according to one study, presumed hospitalrelated transmission of SARS-CoV-2 is suspected in up to $41 \%$ of patients (9). Therefore, to minimize cross-infection and hospital spread of this disease adequate awareness of infection prevention and control (IPC) among medical personnel is imperative.

Infection control procedures include administrative logistic arrangements, environmental hygiene, correct work practice and appropriate use of personal protective equipment (PPE). Prompt detection and effective triage and isolation of potential COVID-19 patients are also essential to prevent unnecessary exposure among patients, healthcare personnel and visitors at the hospital. All healthcare facilities must ensure that their personnel are correctly trained and capable of implementing infection control procedures within a short time frame. Individual healthcare personnel should ensure they understand and adhere to strict infection control requirements (10). An effective and easy-to-follow IPC training program that can be accessed off-site to avoid crowd gathering becomes an invaluable tool. As the official pediatric clinical quality control center in Shanghai, we designed an online initiative for health care workers (HCW) during the early phase of the pandemic in order to enhance their knowledge and skills in various aspects of IPC in the context of COVID-19. In this study, we evaluated the awareness of $\mathrm{HCW}$ on knowledge and practice of infection protection and control following implementation of this initiative. We presented the following article in accordance with the SURGE reporting checklist (available at http:// dx.doi.org/10.21037/tp-20-247).

\section{Methods}

\section{Design of training program}

We developed a 12 -minute training video on essential infection protection and control measures. The materials were put together by experts within the Shanghai Pediatric Clinical Quality Control Center (SPQCC), an organization that focuses on improving pediatric medical security and quality led by the Shanghai Municipal Health Commission (11). The video put a strong emphasis on the correct use of PPE especially the gowning down part which has been documented to be a high-risk procedure leading to disease transmission (12). The training video is copyrighted by SPQCC and can be accessed online (13).

A written guideline has also been prepared, having taken into account relevant documents from the National Health Commission and the World Health Organization, as well as considering the actual situation in our locality (14). The guideline consisted of information related to COVID-19, appropriate staff behavior during the pandemic, clinical management and logistics when handling COVID-19 patients, and other operational procedures related to infection control (15). This study was reviewed and approved by SPQCC in a statement that no formal ethics approval was required in this particular case in the context of COVID-19 pandemic. A letter from SPQCC was sent to participants to inform them about the significance and use of the questionnaire, and informed consent was deemed to be given after completion of the questionnaire. The study conformed to the provisions of the Declaration of Helsinki (as revised in 2013).

\section{Implementation of training program}

There are a total of 81 public partner hospitals affiliated 
with SPQCC and they were all invited to join this program. The partner hospitals included 4 specialized tertiary children's hospitals and 77 general hospitals (28 tertiary and 49 peripheral in service nature). They were requested to arrange for their HCW to go through the guideline and training video. The training program was run once a week for three consecutive weeks to ascertain that all HCW had completed the training.

\section{Questionnaire survey}

An online anonymous questionnaire comprised of 25 items was developed to assess the awareness of knowledge and practice of infection control among HCW in our partner hospitals. Demographic and professional information recorded included age, gender, professionals (pediatrician, nurse, and administrator), professional ranks (junior, middle grade or senior), and level of hospital (peripheral or tertiary). The questionnaire consisted of two parts and focused primarily on the knowledge of COVID-19 ( 2 items), the knowledge of personal infection protection and control (13 items) and clinical practice (10 items). The first part was multiple choice questions (10 items) and the second consisted of true/false questions (15 items). Four points were given for each correct answer and the total score was 100 (Supplementary file 1). We had used factor analysis to test the structure validity. For the internal validity, we used stratified analysis to control the confounding factors influencing association between professionals and degree of hospitals and scores of IPC training. For external validity, we used the stratified-random sampling methods within each partner hospital of SPQCC. The questionnaire was pre-tested in a convenience sample of $10 \mathrm{HCW}$. The cronbach's alpha was 0.752 showing good reliability of inter items. Factor analysis showed construct validity of three components of knowledge of COVID-19, knowledge of IPC, and practice of IPC. Based on their feedback, minor changes were made to enhance clarity and appropriateness of the questions. The items of online questionnaire were designed 'Must enter' to ensure complete completions.

\section{Study participants and sample selection}

The study population consisted of all personnel working in pediatric healthcare settings engaged in patient care activities and they included pediatricians, nurses and administrators. Latter involved in assisting patient triage, first point of contact to see to patients' requests and provided cleaning and handling of soiled medical supplies or equipments, and they were often in direct contact with potentially contaminated environment.

We used a stratified-random sampling method to select HCW according to three professionals (i.e., pediatricians, nurses and administrators) within each partner hospital of SPQCC. Selected participants were sent an online questionnaire for completion within the same day with no financial or other incentives provided.

\section{Statistical analysis}

We estimated the sample size using Sample Size Calculators (https://www.sample-size.net/sample-size-conf-intervalproportion/). As a descriptive study, a minimum sample size of 328 was required taking into account the expected accuracy of the questionnaire to be $>95 \%$ with total width of the confidence interval of $5 \%$. To ensure at least 3 samples within each stratum of three professionals for 81 hospitals, a total of 729 participants were required in this study to meet the calculated minimum sample size (11). The response rate was calculated by the number of respondents divided by total participants invited to the survey. The non-response data were not included in analysis after comparing the characteristics between respondents and non-respondents. The demographic characteristics of the participants were described as $\mathrm{n}(\%)$, and the scores were described as median with interquartile range (IQR). Chi-square test analyses were used to test differences of proportions between peripheral hospitals and tertiary hospitals. Mann-Whitney $U$ test was used to compare the score difference between different hospitals, and KruskalWallis $H$ test was used to compare score difference between professionals, and the different ranks. Multiple comparisons were tested using Mann-Whitney $U$ test with bonferroni correction. No plan for missing data was required, as participants finished the questionnaire completely. All data were analyzed using SPSS version 22.0. A P value of $<0.05$ was considered statistically significant.

\section{Results}

\section{Demographics}

Of the $1,100 \mathrm{HCW}$ who were invited to take part, 1,062 completed questionnaires were returned, giving a response rate of $96.5 \%$. The difference of percentage of type of hospital and professionals between respondents and non- 
Table 1 Demographics and professional characteristics of respondents*

\begin{tabular}{|c|c|c|c|c|}
\hline & Peripheral hospital $(n=592)$ & Tertiary hospital $(n=470)$ & Total $(n=1,062)$ & $\mathrm{P}$ \\
\hline Pediatrician & $215(36.3)$ & $170(36.2)$ & $385(36.3)$ & \\
\hline Nurse & $216(36.5)$ & $194(41.3)$ & $410(38.6)$ & \\
\hline Administrator & $161(27.2)$ & $106(22.6)$ & $267(25.1)$ & \\
\hline Junior & $258(43.6)$ & $256(54.5)$ & $514(48.4)$ & \\
\hline Middle & $260(43.9)$ & $156(33.2)$ & $416(39.2)$ & \\
\hline Senior & $74(12.5)$ & 58 (12.3) & $132(12.4)$ & \\
\hline
\end{tabular}

*, $\mathrm{n}$ indicated the numbers of respondents and categorical variables were presented as $\mathrm{n}(\%) .{ }^{\ddagger}, \mathrm{HCW}$ (healthcare workers) in China are often classified as "senior", "middle" or "junior" grade according to their skill levels and specialization. For example, doctors with senior title refer to chief physician or associate chief physician, middle grade refer to attending physicians and junior title refer to residents (11).

respondents were not statistically significant (Table S1). The median age of the respondents was 32.4 years and 731 (71.2\%) were female. A total of 385 (36.3\%) pediatricians, $410(38.6 \%)$ nurses and $267(25.1 \%)$ administrators were recruited. Among the respondents, $592(55.7 \%)$ and $470(44.3 \%)$ were from peripheral and tertiary hospital respectively. One hundred thirty-two (12.4\%) responders held senior position, 416 (39.2\%) were middle grade and the remaining were junior colleagues (Table 1). Distribution of different HCW from peripheral and tertiary hospitals were similar except number of junior colleagues from tertiary hospital was significantly greater $(54.5 \%$ vs. $43.6 \%$, $\mathrm{P}=0.001)$.

\section{Knowledge and IPC practice among different professional groups}

The median of total score of pediatricians, nurses, and administrators were 96, 100, and 100 respectively. Pediatricians obtained similar score independent of where they worked $(\mathrm{P}=0.249)$. Nurses in tertiary practice performed better than those in peripheral hospitals (100 vs. 96, $\mathrm{P}=0.041)$. Administrators in peripheral hospitals got higher score than those in tertiary hospital (100 vs. $96, \mathrm{P}=0.046)$. In peripheral hospitals, administrators had higher total score than nurses $(100$ vs. $96, \mathrm{P}=0.027)$ and pediatricians (100 vs. 96, $\mathrm{P}=0.024)$. Whereas, there was no statistical difference in total score among professionals from tertiary hospitals and in all responders. And there was no statistically significant difference in total score of different professional ranks between peripheral and tertiary hospitals, and within the same hospital category (Table 2).

The questionnaire focused on three categories, namely knowledge of COVID-19 (2 items), knowledge of IPC (13 items) and clinical practice of IPC (10 items). Table 3 shows the 25 items, sub-categories and percentage of accurate response. The accuracy of the items concerned with knowledge COVID-19 was high (96.0-98.6\%). Likewise, for items that focused on hand hygiene (96.5-99.6\%). Lower scores were seen on questions about protective clothing and mask, protection in out-patient settings and knowledge of high-risk procedures.

Considering scores for individual items, the median with IQR for knowledge on COVID-19 was high $\{8[8,8]\}$. No differences between hospitals, professionals and ranks were demonstrated (Table 4).

The median score for knowledge of IPC was 52 (maximum score for this part). There was no significant difference among the scores of pediatricians, nurses and administrators. Middle grade $\mathrm{HCW}$ in both peripheral and tertiary hospitals were significantly more likely to obtain maximum score than their juniors (Table 5).

The median score for practice of IPC from the responders was $40[36,40]$. There was no significant difference among scores of pediatricians, nurses and administrators. Middle grade HCW in both peripheral and tertiary hospitals got statistically significant higher score than their junior counterparts. In peripheral hospitals, there was a trend where middle-ranked HCW obtained higher score than their seniors. Among all responders, middle grade HCW out-performed both their juniors and seniors in this category (Table 6). 
Table 2 Total score of IPC knowledge and practice, stratified comparison between professionals*

\begin{tabular}{|c|c|c|c|c|}
\hline & Peripheral hospital $(n=592)$ & Tertiary hospital $(n=470)$ & Total $(n=1,062)$ & $\mathrm{P}$ \\
\hline \multicolumn{5}{|l|}{ Professionals } \\
\hline Pediatrician & $96[88,100]$ & $100[92,100]$ & $96[88,100]$ & 0.249 \\
\hline Nurse & $96[84,100]$ & $100[91,100]$ & $100[88,100]$ & 0.041 \\
\hline$P_{\text {professionals }}$ & 0.012 & 0.341 & 0.323 & \\
\hline$P_{\text {pediatricians vs. nurses }}$ & 1.000 & 1.000 & 1.000 & \\
\hline$P_{\text {pediatricians vs. administrators }}$ & 0.024 & 1.000 & 0.372 & \\
\hline$P_{\text {nurses vs. administrators }}$ & 0.027 & 0.453 & 0.885 & \\
\hline Middle & $96[88,100]$ & $100[92,100]$ & $100[88,100]$ & 0.230 \\
\hline Senior & $96[88,100]$ & $98[84,100]$ & $96[88,100]$ & 0.887 \\
\hline $\mathrm{P}_{\text {professional rank }}$ & 0.685 & 0.596 & 0.550 & \\
\hline
\end{tabular}

${ }^{\star}, \mathrm{n}$ indicated the numbers of respondents and the score was presented as median [IQR]. IPC, infection prevention and control.

\section{Discussion}

SARS-CoV-2 has caused of an unusual cluster of viral pneumonia in the world. This situation has rapidly evolved into a pandemic as declared by the WHO on March 11, 2020 (16). It is well-established that HCW are at high risk for both acquiring SARS-CoV-2 from patients and transmitting it to patients due to their close proximity, and may contribute to hospital-related COVID-19 outbreaks (17). A strength of our study is therefore to focus on the effectiveness timely of the point-of-care training program on COVID-19 IPC for PHW by the large sample size of multicenter, cross-sectional questionnaire survey in a city to get good generalizability. Our results documented very good knowledge and practice of infection control among healthcare workers in our partner hospitals. Overall, middle grade colleagues had better performance than other ranks in various aspects of IPC measures.

HCW are expected to play a leadership role in disseminating targeted preventive strategies and knowledge to the public. However, previous work on their awareness of risks has dominantly been based on attitudes rather than knowledge and practical skills (17-19), so we conducted this questionnaire-based survey to evaluate the effectiveness of training on different aspects of infection control for COVID-19 among pediatric HCW in Shanghai. We had to come up with a simple training package in the context of COVID-19 within a short period of time during the initial phase of this pandemic. As crowd gathering was strongly advised against, online learning became the only medium for information transfer. To enhance information retention and recall, bite-sized, visually stimulating demonstration and simple to understand messages were developed into a video and guideline. These were distributed quickly to our partner hospitals where they would arrange for their HCW to go through the training within a tight time frame. Time was basically not on our side, total commitment, support from all participating hospitals and a coordinated effort from the center were essential.

The findings revealed good knowledge of information related to COVID-19 among all responders. The result was remarkably better than one observed in a survey that involved non-teaching public hospitals in Italy during epidemics of seasonal influenza (17), and was close to another survey in a teaching hospital in India, which showed that all residents and more than $90 \%$ of nurses knew about transmission modes of influenza (18). Such a high awareness may be due to the increased access to information and knowledge in the last 10 years, including television, internet, scientific journals, and the up-to-date courses with recent advance and latest literatures issued 
Table 3 Each item concerned, category and the accuracy of the answers*

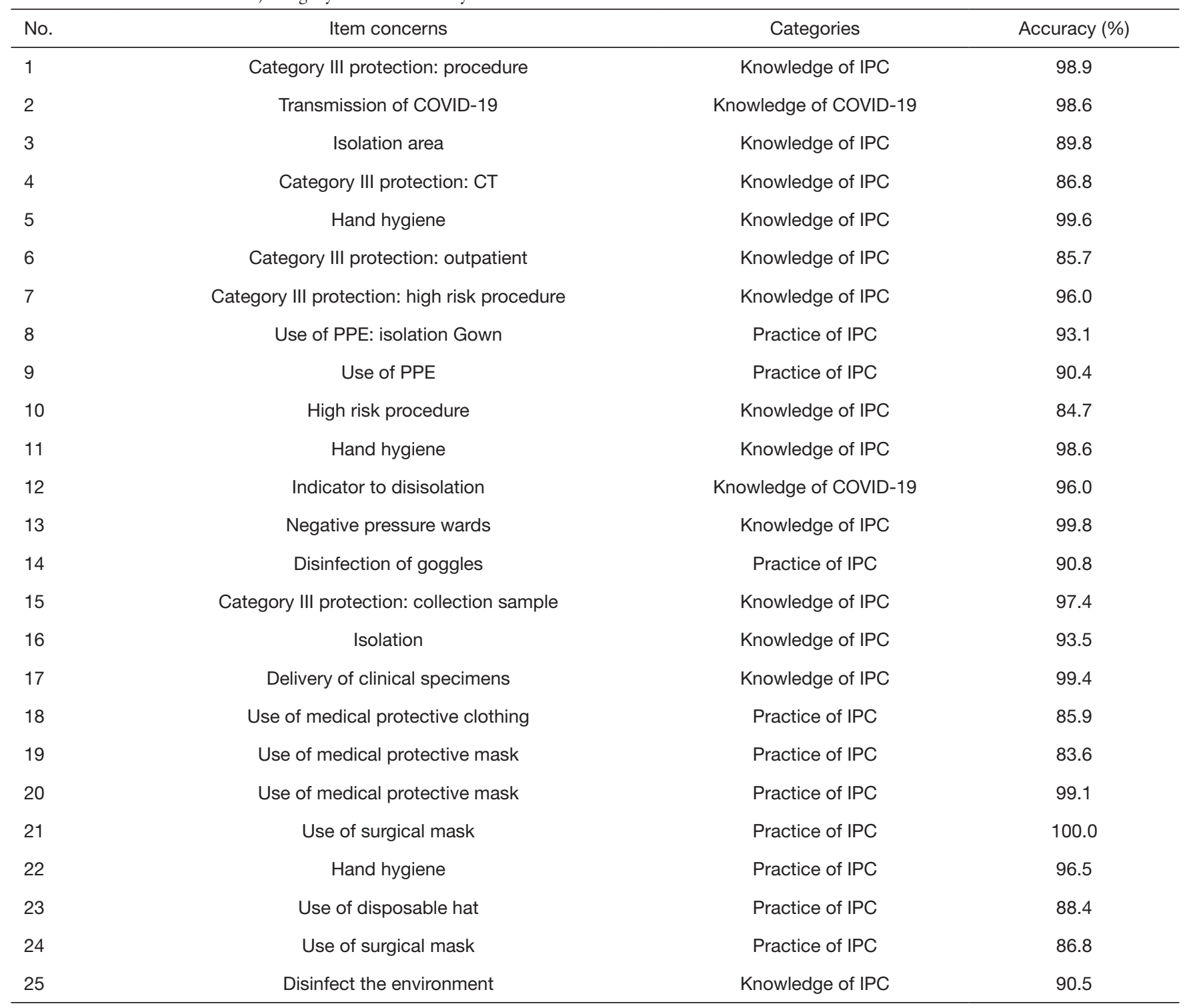

*, No.: number of the item of the questionnaire. IPC, infection prevention and control; COVID-19, coronavirus diseases 2019; CT, computed tomography; PPE, personal protective equipment.

by different healthcare authorities. In our case, we believe the practical and applicable guideline and training video most definitely played a major role in enhancing knowledge among our HCW.

We put a strong emphasis on the importance of hand hygiene in preventing hospital-related infections. Various interventions have been carried out in different healthcare settings in Shanghai, for example through educational programs, providing better hand hygiene products, posting reminders on hospital wards, implementing the supervision mechanism and providing incentives. According to our study, the awareness of hand hygiene among $\mathrm{HCW}$ was very high, ranging from $96.5 \%$ to $99.6 \%$. And no matter what professional position and rank the HCW held, most had very good recognition of hand hygiene. Our result however, was not consistent with other reports (20-22). Zhang's study showed that nurses have a poor hand hygiene adherence in Beijing, ranging from $21.7 \%$ to $30.0 \%$ (23). To some extent, the findings also illustrated the effectiveness of our training program and various interventions implemented in 
Table 4 Knowledge of COVID-19, awareness among healthcare workers ( 2 items, full mark $=8)^{*}$

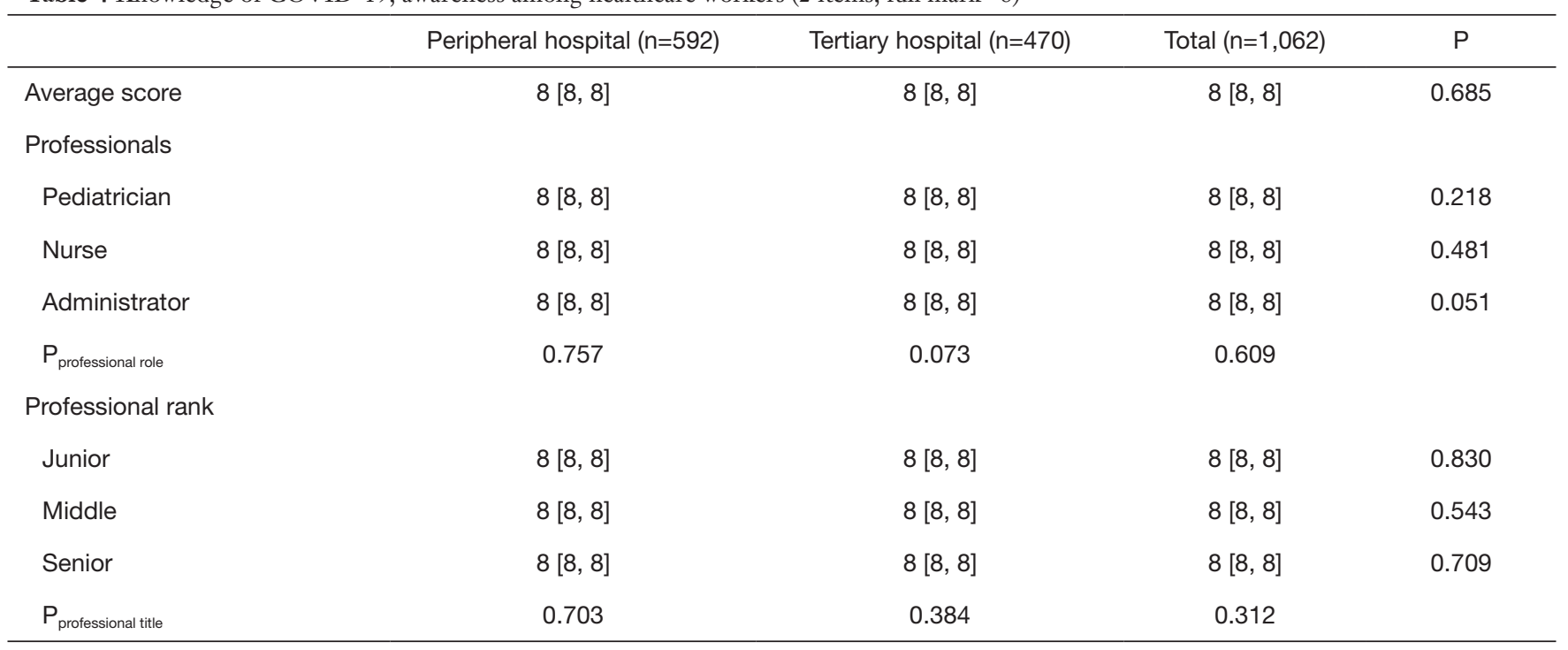

${ }^{*}, \mathrm{n}$ indicated the numbers of respondents and the score was presented as median [IQR]. COVID-19, coronavirus disease 2019.

Table 5 Knowledge of IPC, awareness among healthcare workers (13 items, full mark $=52)^{*}$

\begin{tabular}{|c|c|c|c|c|}
\hline & Peripheral hospital $(n=592)$ & Tertiary hospital $(n=470)$ & Total $(n=1,062)$ & $P$ \\
\hline \multicolumn{5}{|l|}{ Professionals } \\
\hline Pediatrician & $52[48,52]$ & $52[48,52]$ & $52[48,52]$ & 0.220 \\
\hline Nurse & $52[48,52]$ & $52[48,52]$ & $52[48,52]$ & 0.288 \\
\hline$P_{\text {professionals }}$ & 0.448 & 0.441 & 0.912 & \\
\hline \multicolumn{5}{|l|}{ Professional rank } \\
\hline Junior & $52[48,52]$ & $52[48,52]$ & $52[48,52]$ & 0.812 \\
\hline Middle & $52[52,52]$ & $52[52,52]$ & $52[52,52]$ & 0.514 \\
\hline $\mathrm{P}_{\text {junior vs. middle }}$ & 0.006 & 0.003 & $<0.001$ & \\
\hline$P_{\text {junior vs. senior }}$ & 0.378 & 1.000 & 0.381 & \\
\hline $\mathrm{P}_{\text {middle vs. senior }}$ & 1.000 & 0.315 & 0.333 & \\
\hline
\end{tabular}

${ }^{*}, \mathrm{n}$ indicated the numbers of respondents and the score was presented as median [IQR]. IPC, infection prevention and control.

Shanghai in recent years.

The scores of HCW on COVID-19 knowledge, IPC and relevant practice showed no statistically significant difference between workers from peripheral and tertiary hospitals. Although nurses and administrators had different total scores between tertiary and peripheral hospitals, the difference was probably clinically insignificant. Overall all responders had exhibited very good recognition and knowledge base of all areas tested. The intensive and extensive training had shown its beneficial effects among the pediatric medical staff. Children's Hospital of Fudan University is the designated hospital to receive confirmed 
Table 6 Practice of IPC, awareness among healthcare workers (10 items, full mark $=40)^{*}$

\begin{tabular}{|c|c|c|c|c|}
\hline & Peripheral hospital $(n=592)$ & Tertiary hospital $(n=470)$ & Total $(n=1,062)$ & $\mathrm{P}$ \\
\hline \multicolumn{5}{|l|}{ Professionals } \\
\hline Pediatrician & $40[36,40]$ & $40[36,40]$ & $40[36,40]$ & 0.616 \\
\hline Nurse & $40[36,40]$ & $40[36,40]$ & $40[36,40]$ & 0.443 \\
\hline$P_{\text {professional role }}$ & 0.997 & 0.439 & 0.729 & \\
\hline \multicolumn{5}{|l|}{ Professional rank } \\
\hline Junior & $40[32,40]$ & $40[36,40]$ & $40[36,40]$ & 0.543 \\
\hline Middle & $40[40,40]$ & $40[40,40]$ & $40[40,40]$ & 0.739 \\
\hline $\mathrm{P}_{\text {junior vs. middle }}$ & $<0.001$ & 0.021 & $<0.001$ & \\
\hline $\mathrm{P}_{\text {junior vs. senior }}$ & 1.000 & 1.000 & 0.735 & \\
\hline $\mathrm{P}_{\text {middle vs. senior }}$ & 0.051 & 0.252 & 0.009 & \\
\hline
\end{tabular}

${ }^{*}, \mathrm{n}$ indicated the numbers of respondents and the score was presented as median (IQR). IPC, infection prevention and control.

COVID-19 cases and at the time of writing we have admitted 18 patients. Though a small number of cases, COVID-19 is highly infectious, so far none of our HCW has been infected as a result of hospital spread of disease.

Middle grade professional rank out-performed others in both knowledge and practice of IPC. HCW in China are often classified as "senior", "middle" or "junior" grade according to their skill levels and specialization. As expected, middle grade HCW are often the backbone and main personnel in-charge in clinical activities and they are much more involved in daily clinical routines than senior colleagues, who are usually more administrative orientated. Middle grade colleagues also take on a supervisory role to their junior colleagues. Thus, they are more up-todate with current disease pattern, hot medical topics and it does not therefore come as a surprise that this group of personnel should achieve very high score in this exercise. In fact, many of them might have gone through SARS and are more familiar with IPC measures. Junior colleagues have shorter work experience and limited academic and technical abilities. Our findings underscore the importance to target educational activities to junior colleagues to ascertain their knowledge base would be on par with others.

There are certain limitations in this study. We never conducted a pre-training survey to assess knowledge base of our HCW. There is a possibility that their good knowledge and recognition of IPC had nothing to do with our training program. This may be unlikely as our survey assessed areas related specifically to COVID-19 and topics covered by the training program. Our current study design did not allow assessment of longer-term knowledge retention and recall. Disease transmission and patient management can be rather different from one disease to another, thus the same program may not be applicable to all emergent conditions. It is more important to be able to respond quickly and design the most appropriate training materials for sharing within a short time frame. In fact, repeated exposure to IPC measures will more likely to encourage retention.

\section{Conclusions}

A targeted online training program put together by a concerted effort had played a significant part to ensure good knowledge of IPC of our HCW and could have contributed to zero infection among HCW caring for COVID-19 cases. Junior colleagues should be specifically earmarked in educational activities to raise their awareness in dealing with any form of emergent diseases. 


\section{Acknowledgments}

Considerable thanks to the Shanghai Pediatric Clinical Quality Control Center and the participants of this study for their valuable contributions and their time.

Funding: This work was supported by Shanghai Science and Technology Committee [grant number 18411951700], and by Intelligent Medical Research Project of Shanghai Health and Family Planning Commission [grand number: 2018ZHYL0225].

\section{Footnote}

Reporting Checklist: The authors have completed the SURGE reporting checklist. Available at http://dx.doi. org/10.21037/tp-20-247

Data Sharing Statement: Available at http://dx.doi. org/10.21037/tp-20-247

Availability of Data and Materials: The data collected and analyzed during the current study are available from the corresponding author on reasonable request.

Conflicts of Interest: All authors have completed the ICMJE uniform disclosure form (available at http://dx.doi. org/10.21037/tp-20-247). The authors have no conflicts of interest to declare.

Ethical Statement: The authors are accountable for all aspects of the work in ensuring that questions related to the accuracy or integrity of any part of the work are appropriately investigated and resolved. This study was reviewed and approved by Shanghai Pediatric Clinical Quality Control Center (SPQCC) in a statement that no formal ethics approval was required in this particular case in the context of COVID-19 pandemic. A letter from SPQCC was sent to participants to inform them about the significance and use of the questionnaire, and informed consent was deemed to be given after completion of the questionnaire. The study conformed to the provisions of the Declaration of Helsinki (as revised in 2013).

Open Access Statement: This is an Open Access article distributed in accordance with the Creative Commons Attribution-NonCommercial-NoDerivs 4.0 International License (CC BY-NC-ND 4.0), which permits the noncommercial replication and distribution of the article with the strict proviso that no changes or edits are made and the original work is properly cited (including links to both the formal publication through the relevant DOI and the license). See: https://creativecommons.org/licenses/by-nc-nd/4.0/.

\section{References}

1. Lu H, Stratton CW, Tang YW. Outbreak of pneumonia of unknown etiology in Wuhan China: the mystery and the miracle. J Med Virol 2020;92:401-2.

2. Li Q, Guan X, Wu P, et al. Early transmission dynamics in Wuhan, China, of novel coronavirus-infected pneumonia. N Engl J Med 2020;382:1199-207.

3. Huang C, Wang Y, Li X, et al. Clinical features of patients infected with 2019 novel coronavirus in Wuhan, China. Lancet 2020;395:497-506.

4. Holshue ML, DeBolt C, Lingdquist S, et al. First case of 2019 novel coronavirus in the United State. N Engl J Med 2020;382:929-36.

5. Lai CC, Shih TP, Ko WC, et al. Severe acute respiratory syndrome coronavirus 2 (SARS-CoV-2) and coronavirus disease-2019 (COVID-19): The epidemic and the challenges. Int J Antimicrob Agents 2020; 55:105924.

6. Available online: https://covid19.who.int/

7. Wax RS, Christian MD. Practical recommendations for critical care and anesthesiology teams caring for novel coronavirus (2019-nCoV) patients. Can J Anesth 2020;67:568-76.

8. Available online: http:// www.xinhuanet.com/ english/2020-02/02/c_138749620.htm

9. Wang D, Hu B, Hu C, et al. Clinical characteristics of 138 hospitalized patients with 2019 novel coronavirus-infected pneumonia in Wuhan, China. JAMA 2020;323:1061-9.

10. Available online: https://www.cdc.gov/coronavirus/2019ncov/hcp/index.html

11. Mei M, Xu H, Wang LB, et al. Current practice and awareness of pediatric off-label drug use in Shanghai, China-a questionnaire-based study. BMC Pediatrics 2019;19:281.

12. Wolfensberger A, Clack L, Kuster S, et al. Transfer of pathogens to and from patients, healthcare providers, and medical devices during care activity-a systematic review and meta-analysis. Infect Control Hosp Epidemiol 2018;39:1093-107.

13. Available online: https://mp.weixin.qq.com/s/ zhYFIHJNQ3euFNmWRneY2A

14. Jin YH, Cai L, Cheng ZS, et al. A rapid advice guideline for the diagnosis and treatment of 2019 novel coronavirus 
(2019-nCoV) infected pneumonia (standard version). Mil Med Res 2020;7:4.

15. Zhang XB, Wang CQ, Zhu QR, et al. Guiding opinions on the quality control of pediatrics in Shanghai about the prevention and control of COVID-19 (Version 1.0). J Fudan University 2020;47:161-9.

16. Available online: https://www.who.int/dg/speeches/detail/ who-director-general-s-opening-remarks-at-the-mediabriefing-on-covid-19---11-march-2020

17. Albano L, Matuozzo A, Marinelli P, et al. Knowledge, attitudes and behavior of hospital health-care workers regarding influenza $\mathrm{A} / \mathrm{H} 1 \mathrm{~N} 1$ : a cross sectional survey. BMC Infect Dis 2014;14:208.

18. Puri S, Singh A, Koushal V, et al. Knowledge, attitude and practice regarding the H1N1 pandemic amongst healthcare providers, and preparedness in a multispeciality teaching hospital in North India. Public Health 2011;125:795-8.

19. Edeghere O, Fowler T, Silson F, et al. Knowledge,

Cite this article as: Ye Y, Shi P, Gui Y, Li AM, Huang G, Xu H, Lu Q, Hong J, Gu Y, Hu X, Liu G, Wang C, Huang Q, Zhang X. Point-of-care training program on COVID-19 infection prevention and control for pediatric healthcare workers: a multicenter, cross-sectional questionnaire survey in Shanghai, China. Transl Pediatr 2021;10(1):44-53. doi: 10.21037/ tp-20-247 attitudes, experience and behavior of frontline health care workers during the early phase of 2009 influenza A (H1N1) pandemic, Birmingham, UK. J Health Serv Res Policy 2015;20:26-30.

20. Zhao Q, Yang MM, Huang YY, et al. How to make hand hygiene interventions more attractive to nurses: A discrete choice experiment. PLoS One 2018;13:e0202014.

21. Liu WI, Liang SY, Wu SFV, et al. Hand hygiene compliance among the nursing staff in free-standing nursing homes in Taiwan: A preliminary study. Int J Nurs Pract 2014;20:46-52.

22. Chen JK, Wu KS, Lee SS, et al. Impact of implementation of the World Health Organization multimodal hand hygiene improvement strategy in a teaching hospital in Taiwan. Am J Infect Control 2016;44:222-7.

23. Zhang S, Kong X, Lamb KV, et al. High nursing workload is a main associated factor of poor hand hygiene adherence in Beijing, China: An observational study. Int J Nurs Pract 2019;25:e12720. 


\section{Supplementary File 1 Test for correct use of prevention and control supplies during COVID-19 outbreak}

Hospital Professional role Professional title

Gender Age

\section{Part I. Choice questions (1-8 single, 9-10 multiple)}

1. Which of the following operations required Level 3 protection when performed for children with suspected COVID-19?
A. Intravenous injection
B. Temperature measurement
C. Nasopharyngeal swab
D.Physical examination

2. Which of the following is not the established mode of transmission of SARS-Cov-2?
A. Droplet transmission
B. Contact transmission
C. Aerosol transmission
D.Fecal-oral transmission

3. About setting requirements for isolation ward, which of the following is wrong?

A. The clean area, semi-polluted area and polluted area should be marked by red, yellow and green respectively.

B. Space or physical separation should be provided from other medical areas.

C. Set up clean channel, semi-polluted channel, and polluted area channel.

D.No crossing shall be allowed among the three zones (clean zone, semi-polluted zone, polluted zone) and the three channels (clean channel, semi-polluted channel, polluted channel).

4. Which level of protection should be given to medical technicians in the CT room when perform CT examination for children with suspected COVID-19?

A. Basic protection (work clothes, ordinary surgical mask)

B. Level 1 protection (work clothes, surgical mask, disposable hat, disposable gloves, goggles, isolation clothing)

C. Level 2 protection (work clothes, medical protective masks, disposable hats, disposable gloves, goggles, medical protective clothing, shoe covers)

D.Level 3 protection (work clothes, medical protective masks, disposable hats, disposable gloves, fully protective respiratory masks, medical protective clothing, shoe covers)

5. Which of the following moments requires hand hygiene?
A. Before and after patient contact
B. Before aseptic technique
C. After body fluid exposure risk
D. After contact with patient surroundings
E. All of the above are true 
6. The recommended level of personal protection for health care personnel engaged in general clinical practice in contaminated areas of fever outpatients is

A. Level 1 protection (+2) (work clothes, surgical mask, hat, gloves, medical isolation clothing)

B. Level 2 protection (work clothes, medical mask, hat, gloves, medical protective clothing, shoe covers, goggles/mask)

C. Level 3 protection (work clothes, medical protective masks, hats, gloves, medical protective clothing, shoe covers, fully protective respirator/fully protective electric air supply filter respirator)

D. Level 1 protection (+1) (work clothes, surgical mask, hat, gloves)

7. The level of personal protection recommended by medical personnel for outpatient and emergency operations (endotracheal intubation, bronchoscopy, etc.) in general diagnosis and treatment areas is

A. Level 1 protection $(+4)$ or $(+3)$ (work clothes, surgical mask/surgical mask, cap, gloves, medical isolation clothing, goggles/mask)

B. Level 1 protection (1) or (+1) (work clothes, surgical mask, hat, gloves)

C. Level 1 protection (1) (work clothes, surgical mask, cap)

D. Basic protection (work clothes, ordinary surgical mask)

8. The first step to unclogging isolation gown is

A. Unfasten the belt of the isolation garment, tie a slipknot in the front

B. Take off the gloves

C. Wear gloves

D. Unlock the collar

9. Which of the following is true:

A. Remove protective equipment gently to avoid aerosol exposure.

B. When taking off, pay attention to the skin not to touch the contaminated surface to prevent skin exposure.

C. Hand hygiene should be carried out at every step of taking off the protective equipment. Wash hands again after removing all protective equipment.

D. Taking off the protective clothing area from high pollution level to low level, do not reverse.

E. Disposable items should be placed in yellow clinical waste collection bags for centralized disposal.

10. Which of the following are aerosol-prone or high-risk operations?
A. Endotracheal intubation
B. Eye examination
C. Gastroscopy/colonoscopy and sampling
D. Surgery/debridement
E. Medical waste disposal

\section{Part II. True or false questions}

1. Wearing disposable latex gloves when operating can replace hand hygiene for medical staff.

2. The confirmed case of COVID-19 must have a normal body temperature for 3 consecutive days, significantly improved respiratory symptoms, lung imaging evidence of significant absorption of pneumonia, and twice consecutive negative nasopharyngeal swab nucleic acid tests ( 24 hours between the two tests) before the isolation can be lifted.

3. Negative pressure in the negative-pressure-ward in use should be recorded daily.

4. After each wearing of the goggles, it is necessary to use the hydrogen peroxide disinfectant to spray for disinfection comprehensive for 1 minute, then wipe the disinfectant thoroughly and dry them for later use.

5. Level 3 protection is required for handling respiratory specimens of children with suspected or confirmed COVID-19.

6. Confirmed cases of COVID-19 should be isolated in a single room.

7. All specimens of suspected and confirmed cases of COVID-19 should be placed in special biosafety transfer boxes for 
transportation.

8. When wearing protective clothing, the inner gloves are worn outside the cuffs of the protective clothing.

9. When removing a medical protective mask, first pull down the rubber band above and then pull down the rubber band below.

10. When wearing a medical protective mask, air leakage tests must be performed to ensure that the mask is properly worn.

11. When wearing a surgical mask, turn the protective surface (blue) of the mask outwards, with the metallic side facing upward. The mask should completely cover the mouth, nose and chin. Using two index fingers to press the metal plate along the bridge of the nose to make the mask close to the face.

12. After removing the mask and protective clothing, it is necessary to immediately disinfect the hands wearing gloves.

13. When wearing a disposable medical hat, move the elastic side forward, taking care to arrange the hair into the hat.

14. When removing a surgical mask, fold the outer side of the mask outwards, wrap it around and discard it.

15. The disinfection for general outpatient and emergency, indoor floor in wards (excluding neonatal department, NICU, PICU, CCU, hematology department, hemodialysis room and other departments with high risk of infection) should use $1,000 \mathrm{mg} / \mathrm{L}$ chlorine-containing preparations swabbing $>10$ minutes, at least once/day, cleaning and disinfection at any time in case of contamination.

Table S1 Comparison of characteristics between respondents and non-respondents

\begin{tabular}{lccc}
\hline Variables & Respondents & Non-respondents & Total \\
\hline Number of participants & 1062 & 38 & 1100 \\
Type of hospital, $\mathrm{n}(\%)$ & & & \\
Peripheral hospital & $592(55.7)$ & $25(65.8)$ & $617(56.1)$ \\
Tertiary hospital & $470(44.3)$ & $13(34.2)$ & $483(43.9)$ \\
Professionals, $\mathrm{n}(\%)$ & & & $405(36.8)$ \\
Pediatrician & $385(36.3)$ & $20(52.6)$ & $422(38.4)$ \\
Nurse & $410(38.6)$ & $12(31.6)$ & $273(24.8)$ \\
Administrator & $267(25.1)$ & $6(15.8)$ & 209 \\
\hline
\end{tabular}

